\title{
Modelling and Multi-Criteria Decision Making for Selection of Growth Rate Models of Batch Whey Cultivation by Kluyveromyces marxianus var. lactis MC 5
}

\author{
Mitko Petrov
}

Institute of Biophysics and Biomedical Engineering, Bulgarian Academy of Sciences Sofia 1113, Bulgaria, 105 Acad. George Bonchev St.

Email: mpetrov@biomed.bas.bg

\begin{abstract}
This study is focussed on modelling and Multi-criteria Decision Making (MCDM) for selection of growth rate models of batch cultivation by the strain Kluyweromyces marxianus var. lactis MC 5. Different growth rate models Monod, Mink, Tessier, Moser, Aiba, Andrews, Haldane, Luong, Edward, and HanLevenspiel have been considered in order to explain the cell growth kinetics from the basic energetic substrates - lactose and oxygen. The research describes the application of the Preference Ranking Organization Method for Enrichment Evaluations (PROMETHEE) to evaluate and select it from a variety growth rate models. By using the PROMETHEE II method the most suitable for the growth rate model that depends on both of the basic substrates is the Haldane model. Thus, it will be used for modelling the processes.
\end{abstract}

Keywords: multi-criteria decision making, PROMETHEE methods, whey cultivation, growth rate models, Kluyveromyces marxianus var. lactis MC5 strain.

\section{Introduction}

Biotechnological processes are one of the fast-developed strategic fields of science and practice and have a great advance in recent years. Due to their multidisciplinary nature and enormous capabilities they have been applied by microbiologists, biochemists, molecular biologists, bioengineers, chemical engineers, food and pharmaceutical chemists, mathematicians and a whole range of other scientists. These processes are known to be with complicated structure of organization and interdependent characteristics, which determine their non-linearity and nonstationary properties. Therefore, mathematical modelling design, optimization and high-quality control of the underlying processes are very complex, rather time 
consuming and costly tasks. The modelling of bioprocess is a very important process through which the radical principles of microbial synthesis can be discovered. Through dynamics of biotechnological process it can be described by using a balance equation how to apply the radical parameters of a process: cell density, substrate concentration, profitable product, oxygen concentrations, temperature, $\mathrm{pH}$ and all in time [1].

The main objective of Multi-Attribute Decision Making (MADM) is to select the alternative that has the highest score according to the set of the evaluation criteria [2]. A number of methods for solving MADM problems are known up to now. Some of them are Analytic Hierarchy Processes (AHP) [3], Elimination and Choice Expressing Reality (ELECTRE) [4], Multi-Attribute Utility Theory (MAUT) [5], Preference Ranking Organization Method for Enrichment Evaluations (PROMETHEE) [6], etc.

A lot of successful implementations of the PROMETHEE method to various fields are evident, and as such, these methods have found their place in banking, investments, medicine, chemistry, tourism, etc. [7].

More than 15 years in our investigation for modelling, optimization and optimal control of batch and fed-batch whey cultivations by strain Kluyveromyces marxianus var. lactis MC 5 we have used the Mink model for formation of the biomass from the lactose and the Haldane model from oxygen with or without of a mass-transfer in the bioreactor [8-15].

The aim of this study is to view a selection of models for formation of the biomass from lactose and oxygen, and based on the results it offers a new model for the specific growth rate of cultivations of the strain Kluyweromyces marxianus var. lactis MC 5 is.

\section{Materials and methods}

\subsection{Kinetic model}

The batch model of the processes includes the dependence between concentrations of the basic energetic substrates lactose and oxygen, and the cell mass concentration. The model is described by a perfect mixing in a bioreactor [11-15]:

$$
\begin{aligned}
\frac{d X}{d t} & =\mu(S, C) X \\
\frac{d S}{d t} & =-\frac{\mu(S, C)}{Y_{X / S}} X \\
\frac{d C}{d t} & =-\frac{\mu(S, C)}{Y_{X / C}} X+O T R
\end{aligned}
$$

where: $t$ - process time, $\mathrm{h} ; X$ - biomass concentration, $\mathrm{g} / \mathrm{L} ; S$ - lactose concentration, $\mathrm{g} / \mathrm{L} ; C$ - oxygen concentration, $\mathrm{g} / \mathrm{L} ; \mu(S, C)$ - specific growth rate of the cell from lactose and oxygen, $\mu(S, C)=\mu(S) \mu(C), \mathrm{h}^{-1} ; Y_{X / S}, Y_{X / C}-$ yield 
coefficients of formation of the biomass from the lactose and the oxygen, $\mathrm{g} / \mathrm{g}$; OTR - oxygen transfer rate, $O T R=\frac{k_{l} a}{\left(1-\varphi_{\mathrm{G}}\right)}\left(C^{*}-C\right), \mathrm{g} / \mathrm{L} / \mathrm{h} ; k_{l} a-$ mass-transfer coefficient, $\mathrm{h}^{-1} ; \varphi_{G}$ - gas hold-up; $C^{*}-$ mean oxygen concentration, $\mathrm{g} / \mathrm{L}$.

The initial conditions are given as follow: $X(0)=0.2 \mathrm{~g} / \mathrm{L}, S(0)=44 \mathrm{~g} / \mathrm{L}$, $C(0)=6.65 \cdot 10^{-3} \mathrm{~g} / \mathrm{L}$, and $C^{*}(0)=C(0)$.

Gas hold-up $\left(\varphi_{G}\right)$, the power input for dispersion systems the gas-liquid $\left(P_{G}\right)$, and the liquid phase $\left(P_{L}\right)$ are determined by the formulas [16]:

$$
\varphi_{G}=\frac{1.98 Q_{G}}{\left(1+40.9 Q_{G}\right)}\left(\frac{P_{G}}{V}\right)^{0.2}, P_{G}=0.21\left(\frac{Q_{G}}{n d^{3}}\right)^{-0.1} P_{L}^{0.8} \text {, and } P_{L}=60.9 \rho n^{3} d^{5} \mathrm{Re}^{-0.4} \text {, }
$$

where: $Q_{G}$ - gas flow rate, $\mathrm{m}^{3} / \mathrm{s} ; n$ - agitation speed, $\mathrm{s}^{-1} ; d$ - impeller diameter, $\mathrm{m}$; $\rho$-density of the cultural media, $\mathrm{kg} / \mathrm{m}^{3} ; R e$ - Reynolds number.

The gas hold-up have the following values $\left(d=58 \times 10^{-3}, n=13.33 \mathrm{~s}^{-1}\right)$ : for $Q_{G}=16.67 \times 10^{-6} \mathrm{~m}^{3} / \mathrm{s}, \varphi_{G}=0.2305$, and for $Q_{G}=33.34 \times 10^{-6} \mathrm{~m}^{3} / \mathrm{s}, \varphi_{G}=0.2634$. The process description is presented in details in [11-15].

\subsection{Growth rate models}

Nearly all engineering processes have physical constraints. For the processes of the lactose $(S)$ and oxygen $(C)$ mass concentration must be positive all the time; otherwise, an unrealistic solution in the identification problem will be obtained. Therefore we have the following:

$$
\begin{aligned}
& g_{1}(t, \mathbf{x})=-S(t) \leq 0 \\
& g_{2}(t, \mathbf{x})=-C(t) \leq 0
\end{aligned}
$$

In addition, here are the following constrains for stoichiometry by the processes:

$$
\begin{gathered}
g_{3}(t, \mathbf{x})=\frac{S(0)-S(t)}{X(t)-X(0)}-\frac{1}{Y_{X / S}} \leq 0 \\
g_{4}(t, \mathbf{x})=\frac{O T R}{X(t)-X(0)}-\frac{1}{Y_{X / C}} \leq 0
\end{gathered}
$$

where: $\mathbf{x}$-vector of estimated parameters in growth rate models, $\mathbf{x}=\left[\mu_{m}, K_{S}, K_{S I}\right.$, $\left.\ldots, Y_{X / S}, Y_{X / C}\right]^{\mathrm{T}}$.

\subsection{Growth rate models}

The models for the growth rate from lactose $\mu(S)$ and from oxygen $\mu(C)$ rate is unknown, so the work investigates a ten unstructured models (Table 1): $\mathrm{M}_{1}-$ Monod, $\mathrm{M}_{2}-$ Mink, $\mathrm{M}_{3}-$ Tessier, $\mathrm{M}_{4}-$ Moser, $\mathrm{M}_{5}-$ Aiba, $\mathrm{M}_{6}-$ Andrews, $\mathrm{M}_{7}$ - Haldane, $\mathrm{M}_{8}-$ Luong, $\mathrm{M}_{9}-$ Edward, and $\mathrm{M}_{10}-$ Han-Levenspiel [17-22]. 
Table 1. Tested growth rate models dependent from lactose or oxygen

\begin{tabular}{|c|c|c|}
\hline Model & $\mu_{i}^{\prime}(S), \quad i=1, \ldots, 10$ & $\mu_{i}^{\prime \prime}(C), \quad i=1, \ldots, 10$ \\
\hline $\mathrm{M}_{1}$ & $\mu_{1}^{\prime}(S)=\frac{\mu_{m} S}{K_{s}+S}$ & $\mu_{1}^{\prime \prime}(C)=\frac{\mu_{m} C}{K_{C}+C}$ \\
\hline $\mathrm{M}_{2}$ & $\mu_{2}^{\prime}(S)=\frac{\mu_{m} S^{2}}{K_{S}+S^{2}}$ & $\mu_{2}^{\prime \prime}(C)=\frac{\mu_{m} C^{2}}{K_{c}+C^{2}}$ \\
\hline $\mathrm{M}_{3}$ & $\mu_{3}^{\prime}(S)=\mu_{m}\left(1-\exp \left(-S / K_{S l}\right)\right)$ & $\mu_{3}^{\prime \prime}(C)=\mu_{m}\left(1-\exp \left(-C / K_{C I}\right)\right)$ \\
\hline $\mathrm{M}_{4}$ & $\mu_{4}^{\prime}(S)=\frac{\mu_{m} S^{\alpha}}{K_{s}+S^{\alpha}}, \alpha>0$ & $\mu_{4}^{\prime \prime}(C)=\frac{\mu_{m} C^{\alpha}}{K_{C}+C^{\alpha}}, \alpha>0$ \\
\hline $\mathrm{M}_{5}$ & $\mu_{5}^{\prime}(S)=\frac{\mu_{m} S}{K_{S}+S} \exp \left(-S / K_{S I}\right)$ & $\mu_{5}^{\prime \prime}(C)=\frac{\mu_{m} C}{K_{c}+C} \exp \left(-C / K_{C I}\right)$ \\
\hline $\mathrm{M}_{6}$ & $\mu_{6}^{\prime}(S)=\frac{\mu_{m} S}{\left(K_{S}+S\right)\left(1+S / K_{S I}\right)}$ & $\mu_{6}^{\prime \prime}(C)=\frac{\mu_{m} C}{\left(K_{C}+C\right)\left(1+C / K_{C I}\right)}$ \\
\hline $\mathrm{M}_{7}$ & $\mu_{7}^{\prime}(S)=\frac{\mu_{m} S}{K_{S}+S+S^{2} / K_{S I}}$ & $\mu_{7}^{\prime \prime}(C)=\frac{\mu_{m} C}{K_{C}+C+C^{2} / K_{C I}}$ \\
\hline $\mathrm{M}_{8}$ & $\mu_{8}^{\prime}(S)=\frac{\mu_{m} S}{K_{S}+S}\left(1-S / S_{m}\right)^{n}$ & $\mu_{8}^{\prime \prime}(C)=\frac{\mu_{m} C}{K_{C}+C}\left(1-C / C_{m}\right)^{n}$ \\
\hline $\mathrm{M}_{9}$ & $\mu_{9}^{\prime}(S)=\frac{\mu_{m} S}{K_{S}+S+\left(1+S / K_{S I}\right)\left(S^{2} / K_{S}\right)}$ & $\mu_{9}^{\prime \prime}(C)=\frac{\mu_{m} C}{K_{C}+C+\left(1+C / K_{C l}\right)\left(C^{2} / K_{C}\right)}$ \\
\hline $\mathrm{M}_{10}$ & $\mu_{10}^{\prime}(S)=\frac{\mu_{m} S\left(1-S / S_{m}\right)^{n}}{S+K_{S}\left(1-S / S_{m}\right)^{m}}$ & $\mu_{10}^{\prime \prime}(C)=\frac{\mu_{m} C\left(1-C / C_{m}\right)^{n}}{C+K_{C}\left(1-C / C_{m}\right)^{m}}$ \\
\hline
\end{tabular}

In Table 1: $K_{C I}$ - inhibition constant for cell growth on oxygen, $\mathrm{g} / \mathrm{L}$; $K_{I}$ - inhibition constants in different models, g/L; $K_{S}, K_{C}-$ Monod saturation constants for cell growth on lactose and oxygen, g/L; $K_{S I}, K_{C I}-$ inhibition constants for cell growth on lactose or oxygen, $\mathrm{g} / \mathrm{L} ; m, n$ - constants in the Luong and the Han-Levenspiel models.

\subsection{Criteria of evaluation of the model parameters}

The mathematical estimation of the model parameters is based on the minimization of some quantities that can be calculated and the estimation of a function of parameters. The least-squares error is commonly employed as a criterion to inspect how close the computed profiles of the state variables come to the experimental observations [23]:

$$
J=\frac{1}{N} \sum_{j=1}^{N}\left(\frac{\left(X_{e}\left(t_{j}\right)-X_{m}\left(t_{j}\right)\right)^{2}}{X_{e \max }^{2}}+\frac{\left(S_{e}\left(t_{j}\right)-S_{m}\left(t_{j}\right)\right)^{2}}{S_{e \max }^{2}}+\frac{\left(C_{e}\left(t_{j}\right)-C_{m}\left(t_{j}\right)\right)^{2}}{C_{e \max }^{2}}\right)
$$


where: $N$ - number of the experiments; $t_{j}$ - time partitions, h; $X_{e}, S_{e}$, and $C_{e}$ - experimental data, $\mathrm{g} / \mathrm{L} ; X_{m}, S_{m}$, and $C_{m}$ - simulated data with the model, $\mathrm{g} / \mathrm{L}$; $X_{\text {emax }}, S_{\text {emax }}$ and $C_{\text {emax }}$ - maximal values of the experimental data, $\mathrm{g} / \mathrm{L}$.

If the constraints in (2) - (5) are not included in the parameter estimation problem (6), unrealistic predicted values may be found, i.e.

$$
\min _{\mathbf{x}} Q=J+\sum_{k=1}^{4} \int_{t=0}^{t_{f}} w_{t k} g_{k}(t, \mathbf{x}) d t
$$

where $\mathbf{x}$ is a vector of the estimated parameters; $t_{f}$ - final time, $t_{f}=12 \mathrm{~h} ; g_{i}(t, \mathbf{x})$ system constrains, $i=1, \ldots, 4 ; w_{t k}$ - the weight of each constrains, $w_{i}=10^{2}$. Such a large weight of each constrains is chosen to make it easier to identify models. If the constraints are satisfied then $g_{k}(t, \mathbf{x})=0$.

\subsection{Criteria for models validation}

$C_{1}=Q-$ criteria of minimization (7), and the following statistical criteria:

1. $C_{2}-$ statistics $\lambda$. The criterion $C_{2}$ was compared to the tabular Fisher coefficient $\left(F_{T}^{\lambda}\right)$ with a degree of freedom $(M, N-2)$. In this way, it was checked whether it met the condition: $C_{2}>F_{T}^{\lambda}(M, N-2)$, where $M=3$;

2. Relative error for kinetics variables $X, S$, and $C$ : $C_{3}=S_{L}^{X} ; C_{4}=S_{L}^{S} ; C_{5}=S_{L}^{C}$;

3. Fisher coefficient (criteria $C_{6}, C_{7}$, and $C_{8}$ ) for the kinetics variables $X, S$, and $C$ : $C_{6}=F_{X} ; C_{7}=F_{S} ; C_{8}=F_{C}$. Similarly, the obtained values of $C_{3}, C_{4}$, and $C_{5}$ were compared with the tabular Fischer coefficient, but for degrees of freedom $F_{T}(N-$ $2, M)$;

4. Experimental correlation coefficient $R^{2}$ for kinetics variables $X, S$, and $C$ : $C_{9}=R_{X}^{2} ; C_{10}=R_{S}^{2}$; and $C_{11}=R_{C}^{2}$. The obtained values of $C_{9}, \mathrm{C}_{10}$, and $C_{11}$ were compared to the tabular correlation coefficient with a degree of freedom $R_{T}^{2}(N-2)$.

Complete formulas of statistical criteria are presented in [24]. The criteria $C_{1}-C_{8}$ had to be minimized and $C_{9}-C_{11}$ had to be maximized.

We have developed algorithm and program on Compaq Visual FORTRAN 90 to determine the parameters in the model (1) - (6), and computing criteria. For solving a nonlinear problem (6), (7) we have used BCPOL with double precision from IMSL Library of COMPAQ Visual FORTAN 90 [25].

\section{Principles of the PROMETHEE methods}

Using MADM approach is related to the construction of the matrix of the alternatives $\mathbf{A}$. In the matrix $\mathbf{A}$ there are $m$ rows $\left\{A_{1}, \ldots, A_{m}\right\}$ and $n$ columns $\left\{C_{1}, \ldots, C_{n}\right\}$. It has the following general form [2-6]: 


$$
\mathbf{A} \equiv \begin{array}{c|ccc} 
& C_{1} & \ldots & C_{n} \\
\hline A_{1} & a_{11} & \ldots & a_{1 n} \\
\ldots & \ldots & \ddots & \ldots \\
A_{m} & a_{1 m} & \ldots & a_{m n}
\end{array}
$$

Every element $a_{i k}$ of matrix A represents an assessment of the value of the $i$ th alternative $\left(A_{i}\right)$ with respect to the $k$-th criterion $\left(C_{k}\right)$. The evaluation of the $i$-th alternative in terms of all criteria is provided by a row vector $\left(a_{i 1}, \ldots, a_{i n}\right)$. The evaluation of all the alternatives in terms of $k$-th criterion is given by a pillar vector $\left(a_{1 k}, \ldots, a_{m k}\right)^{\mathrm{T}}$.

The decision making process by the PROMETHEE II method consists of four steps that are listed hereafter [6]:

Step 1. This step computes for each pair of possible decisions, the value of the preference function $P_{k}\left(A_{i}, A_{j}\right)$ is associated with each criterion $C_{k}$. In most practical cases $P_{k}\left(A_{i}, A_{j}\right)$ there is a function of the deviation $x=a_{i k}-a_{j k}$ i.e. $P_{k}\left(A_{i}, A_{j}\right)=p_{k}\left(a_{i k}-a_{j k}\right)$ where $p_{k}$ is a non-decreasing function, $p_{k}(x)=0$ for $x \leq 0$, and $0 \leq p_{k}(x) \leq 1$, for $x>0$.

A set of six typical preference functions (Table 2) was proposed by Brans \& Mareschal [6]. The simplicity is the main advantage of these preference functions no more than two parameters in each case.

Table 2. Typical Preference functions in PROMETHEE methods

\begin{tabular}{|c|c|}
\hline Type I: Usual criterion & Type II: U-shape criterion \\
\hline$P_{k}\left(A_{i}, A_{j}\right)= \begin{cases}0 & x \leq 0 \\
1 & x>0\end{cases}$ & $P_{k}\left(A_{i}, A_{j}\right)= \begin{cases}0 & x \leq q_{k} \\
1 & x>q_{k}\end{cases}$ \\
\hline Type III: V-shape criterion & Type IV: Level criterion \\
\hline$P_{k}\left(A_{i}, A_{j}\right)= \begin{cases}0 & x \leq 0 \\
x / p_{k} & 0 \leq x \leq p_{k} \\
1 & x>p_{k}\end{cases}$ & $P_{k}\left(A_{i}, A_{j}\right)= \begin{cases}0 & x \leq q_{k} \\
0.5 & q_{k}<x \leq p_{k} \\
1 & x>p_{k}\end{cases}$ \\
\hline Type V: V-shape with indifference area & Type VI: Gaussian criteria \\
\hline$P_{k}\left(A_{i}, A_{j}\right)= \begin{cases}0 & x \leq q_{k} \\
\frac{x-q_{k}}{p_{k}-q_{k}} & q_{k}<x \leq p_{k} \\
1 & x>p_{k}\end{cases}$ & $P_{k}\left(A_{i}, A_{j}\right)= \begin{cases}0 & x \leq 0 \\
1-\exp \left(-x^{2} / 2 \sigma_{k}^{2}\right) x>0\end{cases}$ \\
\hline
\end{tabular}

Step 2. This step consists in aggregating the preference function of all criteria for each pair of possible decisions. A multi-criteria preference index $\pi\left(A_{i}, A_{j}\right)$ of $A_{i}$ over $A_{j}$ can then be defined considering all the criteria: 


$$
\pi\left(A_{i}, A_{j}\right)=\sum_{k=1}^{n} w_{C_{k}} P_{k}\left(A_{i}, A_{j}\right)
$$

The weight $w_{C_{k}}$ represents the weight of criterion $C_{k}$. It is assumed that $w_{C_{k}} \geq 0$ and $\sum_{k=1}^{n} w_{C_{k}}=1$. As a direct consequence, we have

$$
\pi\left(A_{i}, A_{j}\right) \geq 0 \text { and } \pi\left(A_{i}, A_{j}\right)+\pi\left(A_{j}, A_{i}\right) \leq 1
$$

It describes how important it is for the decision-maker to consider the range of values (from the worst to the best possible level) in this criterion ' $i$ ' compared to the corresponding ranges in the other criteria.

This index also takes values between 0 and 1, and represents the global intensity of preference between the couples of alternatives.

Step 3. The third step being the first that concerns the ranking of the possible decisions consists in computing the outranking flows. For each possible decision $A_{i}$, we compute the positive outranking flow $\phi^{+}\left(A_{i}\right)$ and the negative outranking flow $\phi^{-}\left(A_{i}\right)$.

The positive outranking flow of a possible decision $A_{i}$ is computed by the following formula:

$$
\phi^{+}\left(A_{i}\right)=\frac{1}{n-1} \sum_{j=1}^{n} \pi\left(A_{i}, A_{j}\right)
$$

The negative outranking flow of a possible decision $A_{j}$ is computed by the following formula:

$$
\phi^{-}\left(A_{i}\right)=\frac{1}{n-1} \sum_{j=1}^{n} \pi\left(A_{j}, A_{i}\right)
$$

Step 4. Ranking the actions by a total preorder

The positive outranking flow expresses how much each alternative is outranking all the others. The higher $\phi^{+}\left(A_{i}\right)$, the better the alternative. $\phi^{+}\left(A_{i}\right)$ represents the power of $A_{i}$, its outranking character.

The negative outranking flow expresses how much each alternative is outranked by all the others. The smaller $\phi^{-}\left(A_{i}\right)$, the better the alternative. $\phi^{-}\left(A_{i}\right)$ represents the weakness of $A_{i}$, its outranked character.

$$
\phi\left(A_{i}\right)=\phi^{+}\left(A_{i}\right)-\phi^{-}\left(A_{i}\right)
$$

which can easily be used for ranking the actions:

$$
\begin{aligned}
& A_{i} \text { outranking } A_{j}\left(A_{i} P^{(2)} A_{j}\right) \text { iff } \phi\left(A_{i}\right)>\phi\left(A_{j}\right) \\
& A_{i} \text { is indifferent to } A_{j}\left(A_{i} I^{(2)} A_{j}\right) \text { iff } \phi\left(A_{i}\right)=\phi\left(A_{j}\right)
\end{aligned}
$$


This is the PROMETHEE II complete relation. All the actions of $m$ are now completely ranked but this relation is also less informative and realistic because of the balancing effects between outgoing and incoming flows.

\section{Results and discussion}

\subsection{Results from modelling}

The alternatives to the PROMETHEE II method are the growth models from $\mathrm{M}_{1}$ to $\mathrm{M}_{10}$, and criteria from $C_{1}$ to $C_{11}$.

The matrices of alternatives for growth rate from lactose $\left(\mathbf{A}_{\mathbf{1}}\right)$ and oxygen $\left(\mathbf{A}_{2}\right)$ are shown in Table 3.

Table 3. Matrix of the alternatives for formation of biomass from lactose $\mu(S)$ and from oxygen $\mu(C)$

\begin{tabular}{|c|c|c|c|c|c|c|c|c|c|c|c|}
\hline \multicolumn{12}{|c|}{ Matrix of the alternatives $\left(\mathbf{A}_{1}\right)$ for formation of biomass from lactose $\mu(S)$} \\
\hline Model & $C_{1 \times 10^{-3}}$ & $C_{2}$ & $C_{3}$ & $C_{4}$ & $C_{5}$ & $C_{6}$ & $C_{7}$ & $C_{8}$ & $C_{9}$ & $C_{10}$ & $C_{11}$ \\
\hline $\mathrm{M}_{1}$ & 110.370 & 189.515 & 0.342 & 0.686 & 7.513 & 1.012 & 1.233 & 1.030 & 0.976 & 0.950 & 0.857 \\
\hline $\mathrm{M}_{2}$ & 83.018 & 436.561 & 0.276 & 1.980 & 7.148 & 1.053 & 1.219 & 1.086 & 0.999 & 0.965 & 0.918 \\
\hline $\mathrm{M}_{3}$ & 82.092 & 376.796 & 0.308 & 0.464 & 7.476 & 1.073 & 1.006 & 1.106 & 0.998 & 0.960 & 0.900 \\
\hline $\mathrm{M}_{4}$ & 84.640 & 426.878 & 0.263 & 2.133 & 7.486 & 1.045 & 1.243 & 1.098 & 0.999 & 0.967 & 0.916 \\
\hline $\mathrm{M}_{5}$ & 166.163 & 231.067 & 0.422 & 0.698 & 7.193 & 1.023 & 1.190 & 1.057 & 0.980 & 0.927 & 0.803 \\
\hline $\mathrm{M}_{6}$ & 114.108 & 363.709 & 0.398 & 0.543 & 6.529 & 1.096 & 1.001 & 1.080 & 0.993 & 0.938 & 0.877 \\
\hline $\mathrm{M}_{7}$ & 68.712 & 169.286 & 0.378 & 0.782 & 0.753 & 1.153 & 1.286 & 1.105 & 0.894 & 0.965 & 0.970 \\
\hline $\mathrm{M}_{8}$ & & & 0.382 & 0.783 & 0.712 & 1.156 & 1.286 & 1.106 & 0.893 & 0.965 & 0.971 \\
\hline $\mathrm{M}_{9}$ & & 169.141 & 0.382 & 0.783 & 0.722 & & 1.287 & 1.108 & 0.893 & 0.964 & 0.970 \\
\hline $\mathrm{M}_{10}$ & 70.999 & 168.968 & 0.387 & 0.784 & 0.736 & 1.150 & 1.289 & 1.113 & 0.893 & 0.964 & 0.968 \\
\hline \multicolumn{12}{|c|}{ Matrix of the alternatives $\left(\mathbf{A}_{2}\right)$ for formation of biomass from oxygen $\mu(C)$} \\
\hline Model & $C_{1} \times 10^{-3}$ & $C_{2}$ & $C_{3}$ & $C_{4}$ & $C_{5}$ & $C_{6}$ & $C_{7}$ & $C_{8}$ & $C_{9}$ & $C_{10}$ & $C_{11}$ \\
\hline $\mathrm{M}_{1}$ & 13.108 & 173.342 & 0.192 & 0.495 & 0.561 & 1.001 & 1.078 & 1.045 & 0.996 & 0.979 & 0.994 \\
\hline $\mathrm{M}_{2}$ & 10.286 & 150.967 & 0.184 & 0.476 & 0.429 & 1.011 & 1.088 & 1.023 & 0.996 & 0.984 & 0.993 \\
\hline $\mathrm{M}_{3}$ & 14.991 & 204.837 & 0.179 & 0.456 & 0.327 & 1.003 & 1.006 & 1.022 & 0.998 & 0.979 & 0.992 \\
\hline $\mathrm{M}_{4}$ & 10.932 & 153.097 & 0.162 & 0.451 & 1.100 & 1.014 & 1.074 & 1.040 & 0.996 & 0.985 & 0.988 \\
\hline $\mathrm{M}_{5}$ & 18.308 & 210.849 & 0.207 & 0.469 & 0.418 & 1.011 & 1.009 & 1.038 & 0.998 & 0.977 & 0.989 \\
\hline $\mathrm{M}_{6}$ & 14.786 & 206.348 & 0.174 & 0.458 & 0.319 & 1.000 & 1.005 & 1.014 & 0.998 & 0.979 & 0.993 \\
\hline $\mathrm{M}_{7}$ & 10.259 & 155.067 & 0.181 & 0.488 & 0.338 & 1.005 & 1.088 & 1.010 & 0.996 & 0.982 & 0.995 \\
\hline $\mathrm{M}_{8}$ & 15.736 & 214.314 & 0.182 & 0.470 & 0.330 & 1.003 & 1.005 & 1.020 & 0.999 & 0.977 & 0.994 \\
\hline $\mathrm{M}_{9}$ & 14.088 & 176.752 & 0.197 & 0.499 & 0.693 & 1.001 & 1.072 & 1.057 & 0.996 & 0.979 & 0.993 \\
\hline $\mathrm{M}_{10}$ & 15.911 & 210.977 & 0.186 & 0.466 & 0.355 & 1.005 & 1.006 & 1.026 & 0.999 & 0.977 & 0.993 \\
\hline
\end{tabular}


Now, let us see the matrixes $\mathbf{A}_{1}$ and $\mathbf{A}_{2}$. The criteria of evaluation of the model parameters is changed in the interval $C_{1} \in[68.712,166.163]$ for lactose, and in the interval $C_{1} \in[10.259,18.308]$ for oxygen. The relative errors for every kinetic variable $M$ is changed in the interval $\mathrm{C}_{3-5} \in[0.263,7.513]$ for lactose, and $C_{3-5} \in[0.162,1.100]$ for oxygen.

The criteria $C_{2}$ and $C_{6}-C_{11}$ are statistical. They are used for the model validation. The theoretical values of $C_{2}$ and $C_{6}-C_{11}$ are given from statistical tables [26]. Fisher coefficient for $C_{2}$ (Statistic $\lambda$ ) is $F_{(3,10)}^{\lambda}=3.71$. Fisher coefficients $\left(C_{6}-C_{8}\right)$ are $F_{(10,3)}=8.79$, and for correlation coefficients $\left(C_{9}-C_{11}\right)$ the tabular value is $R_{(10)}=0.576$.

The $C_{2}>F_{3,10}^{\lambda}=3.71$, the experimental Fisher coefficients $\left(C_{6}-C_{8}\right)$ are in the interval $C_{6}-C_{8} \in[1.001,1.228]<F_{10,3}=8.79$, and the experimental correlation coefficients $\left(C_{9}-C_{11}\right)$ are in interval $C_{9}-C_{11} \in[0.893,0.999]>R_{(10)}=0.576$. By the presented results it can be noted that in terms of the criteria for validation $\left(C_{2}, C_{6}-C_{11}\right)$ all growth rate models dependent on lactose or oxygen are adequate.

The application of the PROMETHEE II method will determine which of the tested models for lactose and oxygen will be most appropriate.

\subsection{Application of PROMETHEE II method}

The new PROMETHEE-GAIA software named Visual PROMETHEE is developed under the supervision of $B$. Mareschal at VPSolutions [27, 28]. If we want to use Visual PROMETHEE software it is necessary to choose Preference functions and the weights of each criterion. For lactose and oxygen we need to choose equality of Preference functions.

For criteria $C_{1}-C_{5}$ we have chosen Gaussian Preference function with $\sigma_{k}=3.0$ and weight $w_{c_{k}}=0.1364$ for $k=1, \ldots, 5$. For criteria $C_{6}-C_{11}$ we have chosen Usual Preference function with weight $w_{c_{k}}=0.053$ for $k=6, \ldots, 11$ and $\sum_{k=1}^{n} w_{C_{k}}=1$.

For the criteria $C_{1}-C_{5}$ we have chosen Gaussian Preference function, because the same criteria are non-linear functions and they have normal distribution. The selection of usual preference function for the criteria $C_{6}-C_{11}$ follows from the fact that the values of the criteria are near to 1 and they are almost linear functions.

When applying PROMETHEE II method the results for lactose and oxygen are shown in Table 4. 
Table 4. PROMETHEE flow table for lactose and oxygen

\begin{tabular}{|c|c|c|c|c|c|c|c|c|}
\hline Rank & Lactose & $\phi\left(\boldsymbol{A}_{\boldsymbol{i}}\right)$ & $\boldsymbol{\phi}^{+}\left(\boldsymbol{A}_{\boldsymbol{i}}\right)$ & $\boldsymbol{\phi}\left(\boldsymbol{A}_{\boldsymbol{i}}\right)$ & Oxygen & $\boldsymbol{\phi}\left(\boldsymbol{A}_{\boldsymbol{i}}\right)$ & $\boldsymbol{\phi}^{+}\left(\boldsymbol{A}_{\boldsymbol{i}}\right)$ & $\boldsymbol{\phi}\left(\boldsymbol{A}_{\boldsymbol{i}}\right)$ \\
\hline $\mathbf{1}$ & $\boldsymbol{M}_{7}$ & $\mathbf{0 . 2 0 3 8}$ & $\mathbf{0 . 4 1 8 0}$ & $\mathbf{0 . 2 1 4 2}$ & $\boldsymbol{M}_{7}$ & $\mathbf{0 . 2 2 9 2}$ & $\mathbf{0 . 4 0 3 8}$ & $\mathbf{0 . 1 7 4 6}$ \\
\hline 2 & $M_{8}$ & 0.1545 & 0.3851 & 0.2306 & $M_{6}$ & 0.1975 & 0.3605 & 0.1631 \\
\hline 3 & $M_{9}$ & 0.0202 & 0.3256 & 0.3054 & $M_{2}$ & 0.0815 & 0.3164 & 0.2349 \\
\hline 4 & $M_{2}$ & 0.0139 & 0.3436 & 0.3296 & $M_{8}$ & 0.0782 & 0.3184 & 0.2402 \\
\hline 5 & $M_{4}$ & 0.0116 & 0.3539 & 0.3423 & $M_{3}$ & 0.0369 & 0.2909 & 0.2541 \\
\hline 6 & $M_{10}$ & 0.0036 & 0.3241 & 0.3205 & $M_{1}$ & -0.0118 & 0.2676 & 0.2794 \\
\hline 7 & $M_{1}$ & -0.0496 & 0.3301 & 0.3797 & $M_{10}$ & -0.0692 & 0.2309 & 0.3001 \\
\hline 8 & $M_{3}$ & -0.0876 & 0.3001 & 0.3877 & $M_{9}$ & -0.1062 & 0.2233 & 0.3295 \\
\hline 9 & $M_{6}$ & -0.1211 & 0.2936 & 0.4147 & $M_{4}$ & -0.1490 & 0.2564 & 0.4053 \\
\hline 10 & $M_{5}$ & -0.1493 & 0.2825 & 0.4318 & $M_{5}$ & -0.2870 & 0.1492 & 0.4362 \\
\hline
\end{tabular}

The investigation (Table 4) shows the Haldane-Haldane model for specific growth rate $\mu(S, C)$ has showed best results. The mathematical relationships of the model are as follows:

$$
\mu(S, C)=\frac{\mu_{m} S}{\left(K_{S}+S+S^{2} / K_{S I}\right)} \frac{C}{\left(K_{C}+C+C^{2} / K_{C I}\right)}
$$

After an application of the developed algorithm and program for parametric identification in the model (1). The model for specific growth rate (8) they have the following values:

$$
\begin{aligned}
& \mu_{m}=0.808 \mathrm{~h}^{-1}, K_{S}=0.101 \mathrm{~g} / \mathrm{L}, K_{S I}=647.058 \mathrm{~g} / \mathrm{L}, K_{C}=0.979 \times 10^{-3} \mathrm{~g} / \mathrm{L}, \\
& K_{C I}=1.334 \mathrm{~g} / \mathrm{L}, Y_{X / S}=0.441 \mathrm{~g} / \mathrm{g}, \mathrm{Y}_{X / C}=243.555 \times 10^{-3} \mathrm{~g} / \mathrm{g}, k_{l} a=120.12 \mathrm{~h}^{-1} .
\end{aligned}
$$

The criteria $\left(C_{k}, k=1, \ldots, 11\right)$ have the following values:

$$
\begin{aligned}
& C_{1}=9.073 \times 10^{-3}, C_{2}=148.281, C_{3}=0.156 \text {, } \\
& C_{4}=0.473, \quad C_{5}=0.699, \quad C_{6}=1.012, \quad C_{7}=1.081, \\
& C_{8}=1.016, \quad C_{9}=0.996, \quad C_{10}=0.984 \text {, and } C_{11}=0.995 \text {. }
\end{aligned}
$$

The obtained results show that the model is adequate and can be used to modelling of the process.

The results after simulations for the biomass $(X)$, lactose $(S)$ and oxygen $(C)$ for the bath cultivation of Kluyweromyces Marxianus var. lactis MC 5 with model are shown in Fig. 1. 


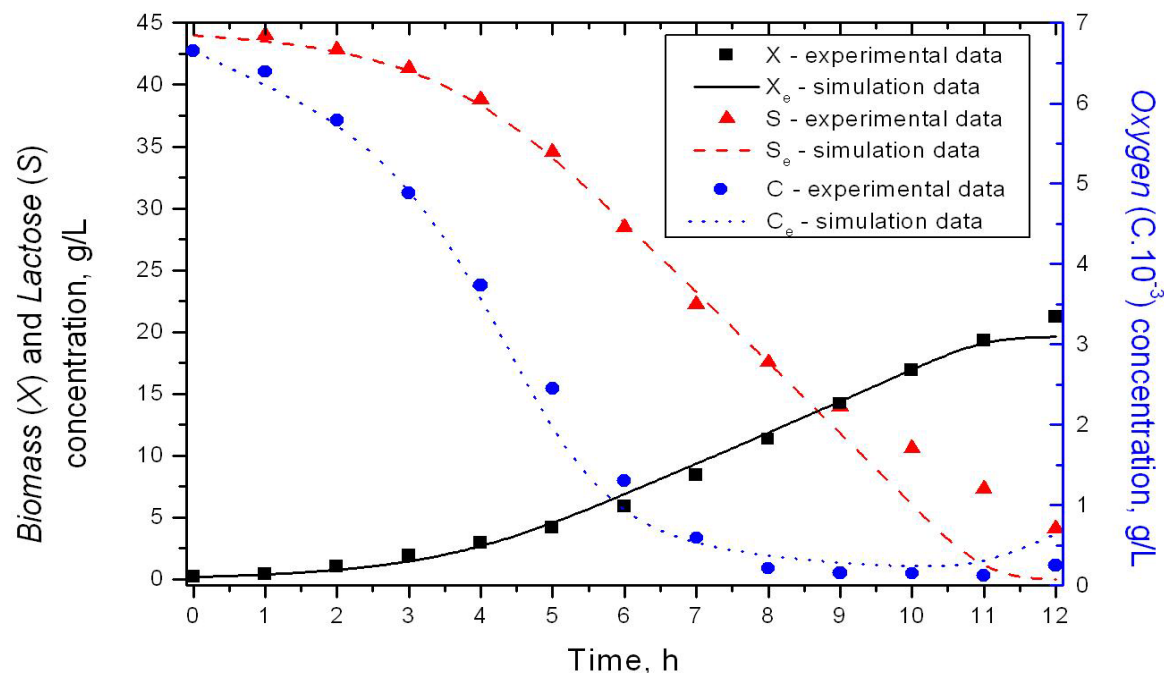

Fig. 1. Simulation of biomass lactose and oxygen concentration of the Kluyweromyces marxianus var. lactis MC 5

The results in Fig. 1 show that the biomass and oxygen describe good model of the process of the model. But this does not refer to the lactose.

\section{Conclusion}

Mathematical models of a batch process of a lactose oxidation from a natural substratum in cultivation of strain Kluyweromyces marxianus var. lactis MC 5 are presented in this study. We have included the gas hold-up in the model of the bioreactor. Then models were investigated for the growth rate from lactose and from oxygen: Monod, Mink, Tessier, Moser, Aiba, Andrews, Haldane, Luong, Edward, and Han-Levenspiel. The statistical results - statistics $\lambda$. Fisher and correlation coefficient for the growth rate from lactose and oxygen showed that all the investigated models are adequate.

Using the PROMETHEE II method shows that the most suitable model for growth rate dependent on both basic substrate is Haldane-Haldane which will be used for modelling of the process.

The specifying of the coefficients in this model will be made in another paper, where the identification will be done simultaneously for the batch and fedbath cultivations by stain Kluyveromyces marxianus var. lactis MC 5. After that this model should be used for modelling, optimisation and optimal control of the batch and fed-batch processes.

\section{References}

1. Viesturs, U., Simeonov, I., Pencheva, T., Vanags, J., Petrov, M., Pavlov, Y., Roeva, O., Ilkova, T., Vishkins, M., Hristozov, I.: Contemporary approaches to modelling, 
optimisation and control of biotechnological processes. Prof. M. Drinov Publishing House of Bulgarian Academy of Sciences, Sofia. Bulgaria (2010).

2. Taha, R., Daim, T.: Multi-criteria applications in renewable energy analysis. A literature review. T. Daim. T. Oliver. J. Kim (Eds.). Research and Technology Management in the Electricity Industry. Springer-Verlag, London, 17-30 (2013).

3. Saaty, R.: The analytic hierarchy process-what it is and how it is used. Math. Modelling 9(3-5), 161-176 (1987).

4. Figueira, J., Mousseau, V., Roy, B.: ELECTRE methods. Greco S. (Ed.) Multiple Criteria Decision Analysis: State of the Art Surveys. vol. 78, London, Springer, 133153 (2005).

5. Dyer, J.: MAUT - Multiatribute utility theory. Greco S. (Ed.). Multiple Criteria: State of the Art Surveys. vol. 78. London. Springer, 265-292 (2005).

6. Brans, J.-P., Mareschal, B.: PROMETHEE methods. Multiple Criteria Decision Analysis: State of the Art Surveys. vol. 78. London. Springer, 163-186 (2005).

7. The PROMETHEE bibliographical database. http://www.prometheegaia.net/bibliographical-database.html. last accessed 2020/01/16.

8. Petrov, M., Ilkova, T., Tzonkov, St.: Modeling and fuzzy optimization of whey fermentation by Kluyveromyces marxianus var. lactis MC 5. Chemical and Biochemical Engineering Quarterly 19(1), 49-55 (2005).

9. Petrov, M.: A Multiple-objective optimization of whey fermentation in stirred tank. International Journal BIOautomation 5, 39-48 (2006).

10. Petrov, M., Ilkova, T: Modeling and optimization of the lactose oxidation for receiving of ecological clean and waste less technology at the white brine cheese production. Ecology - Scientific, art. no. 2, 256-272 (2007).

11. Ilkova, T., Petrov, M.: Rollout optimization of lactose oxidation for receiving of ecological clean and waste less technology at cheese production. Journal of International Scientific Publication: Ecology \& Safety 3(1), 466-475 (2009).

12. Petrov, M., Ilkova, T.: A combined algorithm for multi-objective fuzzy optimization of whey fermentation. Chemical and Biochemical Engineering Quarterly 23(2), 153-160 (2009).

13. Ilkova, T. Petrov., M., Roeva, O.: Neuro-dynamic strategy for obtain an optimal feed profile in a whey bioprocess. In: Proc. of Int. Conference Automatic and Informatics'11 3-7 October 2011, Sofia. Bulgaria, pp. B-109-B-112 (2011).

14. Ilkova, T., Petrov, M, Roeva, O.: Optimization of a whey bioprocess using neurodynamic programming strategy. Biotechnol. \& Biotec. EQ. 26(5) 3249-3253 (2012).

15. Petrov, M., Ilkova, T., Vanags, J.: Modelling of batch whey cultivation by strain Kluyveromyces marxianus var. lactis MC 5 with investigation of mass transfer processes in the bioreactor. International Journal BIOautomation 19(1). Suppl. 1, S81S92 (2015).

16. Petrov, M., Tzonkov, St.: Modelling of mass transfer and optimization of stirred bioreactors. Bioprocess Engineering 21(1), 61-63 (1999).

17. Gera, N., Uppaluri, R. V. S., Sen, S., Venkata Dasuc, V.: Growth kinetics and production of glucose oxidase using Aspergillusniger NRRL326. Chemical and Biochemical Engineering Quarterly 22(3), 315-320 (2008).

18. Kim, D.-J., Choi, J.-W., Choi, N.-C.: Mahendran, B., Lee, C.-E.: Modeling of growth kinetics for pseudomonas spp. during benzene degradation. Applied Microbiology and Biotechnology 69, 456-462 (2005). 
19. Saravanan, P., Pakshirajan, K., Saha, P.: Kinetics of phenol degradation and growth of predominant Pseudomonas species in a simple batch stirred tank reactor. Bulgarian Chemical Communications 43(4), 502-509 (2011).

20. Sudipta, D., Mukherjee, S.: Performance and kinetic evaluation of phenol biodegradation by mixed microbial culture in a batch reactor. International Journal of Water Resources and Environmental Engineering 2(3), 40-49 (2010).

21. Cutin, K., Dutta, D., Venkata, V., Mahanty, P., Anand, A.: Substrate inhibition growth kinetics for cutinase producing Pseudomonas cepacia using tomato-peel extracted. Chemical and Biochemical Engineering Quarterly 29(3), 437-445 (2015).

22. Giridhar, R., Srivastava, A.: Model based constant feed fed-batch L-Sorbose production process for improvement in L-Sorbose productivity. Chemical and Biochemical Engineering Quarterly 14(4), 133-140 (2000).

23. Wang, F.-S., Tzu-Liang, S., Horng-Jhy, J.: Hybrid differential evolution for problems of kinetic parameter estimation and dynamic optimization of an ethanol fermentation process. Industrial \& Engineering Chemistry Research 40, 2876-2885 (2001).

24. Petrov, M., Ilkova, T.: Intercriteria decision analysis for choice of growth rate models of batch cultivation by strain Kluyveromyces marxianus var. lactis MC 5, Journal of International Scientific Publications: Materials, Methods \& Technology 10, 468-486 (2016).

25. COMPAQ Visual FORTRAN Programmer's Guide. v. 6.6. Compaq Computer Corporation. Houston, Texas (2001).

26. Vuchkov, I., Stoyanov, St.: Mathematical Modelling and Optimization of Technological Objects. Technique, Sofia, (in Bulgarian) (1986).

27. PROMETHEE-Gaia software. http://www.promethee-gaia.net/software.html. Last accessed 2019/11/05.

28. Visual PROMETHEE 1.4 Manual. September 5. 2013. 